\title{
Classification Using $\Phi$-Machines and Constructive Function Approximation
}

\author{
DOINA PRECUP \\ School of Computer Science, McGill University, Montreal, QC, H3A 2A7, Canada \\ PAUL E. UTGOFF \\ Department of Computer Science, University of Massachusetts, MA 01003-4610, USA \\ Editor: David Aha
}

dprecup@cs.mcgill.ca

\begin{abstract}
This article presents a new classification algorithm, called CLEF, which induces a $\Phi$-machine by constructing its own features based on the training data. The features can be viewed as defining subsets of the instance space, and they allow CLEF to create useful non-linear functions over the input variables. The algorithm is guaranteed to find a classifier that separates the training instances, if such a separation is possible. We compare CLEF empirically to several other classification algorithms, including a well-known decision tree inducer, an artificial neural network inducer, and a support vector machine inducer. Our results show that the CLEF-induced $\Phi$-machines and support vector machines have similar accuracy on the suite tested, and that both are significantly more accurate than the other classifiers produced. We argue that the classifiers produced by CLEF are easy to interpret, and hence may be preferred over support vector machines in certain circumstances.
\end{abstract}

Keywords: classification, constructive induction, linear machine, $\Phi$-machine, non-linear discrimination, decision tree, support vector machine

\section{Introduction}

A number of issues surround the problem of designing an algorithm that constructs a classifier based on labeled examples of the various classes. Of central interest is the design of an automatic procedure that will yield a classifier that separates the training examples, and generalizes well to new instances in the domain. Many algorithms for classifier induction have been implemented and tested previously. Notable examples include algorithms for inducing decision trees, artificial neural networks of various kinds, linear machines, and support vector machines.

In this article, we present an alternative approach to classifier induction, based on a novel feature construction mechanism. Our algorithm, CLEF, induces a classifier in the form of a $\Phi$-machine (Nilsson, 1965; Duda \& Hart, 1973), which is a multi-class non-linear separator. $\Phi$-machines are an extension of linear machines. They use nonlinear features to map patterns that are otherwise non-separable into a space where they become linearly separable. Traditionally, $\Phi$-machines have been constructed manually by specifying a fixed set of non-linear features, and then training a set of weights that will provide a linear combination of the features. The novelty of our approach consists of constructing the features 
of a $\Phi$-machine automatically, based on the training data, instead of using pre-defined features. Our feature construction method, called ELF, was originally designed for realvalued function approximation (Utgoff \& Precup, 1998). In this article, we incorporate ELF into a $\Phi$-machine inducer in a straightforward way. A $\Phi$-machine consists of at least two real-valued functions, so our approach is to use ELF in order to construct the features of each such function.

Constructive learning methods have been embedded before in other classifier induction algorithms, such as decision tree induction (e.g., Pagallo, 1990; Matheus \& Rendell, 1989; Gamma, 1999; Brodley \& Utgoff, 1995), training of sigmoid neural networks (e.g., Ash, 1989; Fahlman \& Lebiere, 1990; Hanson, 1990) and multi-layer perceptron induction (e.g., Nadal, 1989; Mezard \& Nadal, 1989; Frean, 1990b; Yang, Parekh, \& Honavar, 1999; Parekh, Yang, \& Honavar, 2000). Our motivation for using the $\Phi$-machine framework was based on the advantages that $\Phi$-machines have compared to other classification methods.

For instance, although top-down decision tree induction has many desirable strengths for classifier construction, principally speed and compactness, it partitions the data recursively. ${ }^{1}$ This partitioning creates separate subproblems, each of which is based on fewer of the training examples. It is well known that a reduced amount of training data can lead to less well supported generalization and increased error. This principle was illustrated crisply by Pagallo (1990) in her work on FRINGE. By constructing compound tests on which to partition the data, less partitioning and therefore larger training sets per block typically led to lower error rates (Pagallo \& Haussler, 1990). In contrast with decision trees, $\Phi$-machines compute their weights based on all the training data.

Error back-propagation in artificial neural networks (Rumelhart, Hinton, \& Williams, 1986) also computes a set of parameters based on the entire training set. Based only on this feature of the algorithm, one would expect it to produce classifiers with lower error rates than decision tree induction. However, empirical results (Fisher \& McKusick, 1989; Mooney et al., 1989) show that the error rates of classifiers constructed with these two methods are similar. There are many potential reasons for this result. One reason is that an artificial neural network can become trapped at a local minimum in the error surface over the weight space. Moreover, there is no guarantee that the features found by the hidden layer using backpropagation will be linearly independent. In contrast, the error surface of a $\Phi$-machine has a single global minimum, so local minima are not an issue.

The linear independence of the features is also important for $\Phi$-machines, since it guarantees the convergence of the parameters to a separation of the training data, if such a separation is possible (Duda \& Hart, 1973). When the features of a $\Phi$-machine are hand-crafted, their linear independence has to be ensured by the designer of the system. Our feature construction algorithm is designed to create linearly independent features automatically, thus greatly facilitating the creation of a $\Phi$-machine.

Our algorithm (described in detail in Section 3) associates weights with subsets of the instance space. The features it creates are Boolean descriptions of these subsets, in conjunctive form. The features are generated incrementally, in a general-to-specific order, which allows the construction of fairly compact classifiers. Because the features have a logical form, the resulting classifiers are easy to interpret. 
The article is organized as follows. Section 2 contains a general description of the classifier induction problem. In Section 3, we describe in detail the CLEF decision tree inducer. Section 4 contains an illustration of the output produced by CLEF, and a discussion of the interpretability of the generated classifiers. Section 5 presents an empirical comparison of CLEF with several other machine learning algorithms. Related work is discussed in Section 6. Section 7 contains conclusions and directions for future work.

\section{The classifier induction task}

The task of classifier induction algorithms is to find an approximate definition for an unknown function $f: \mathbf{X} \rightarrow\left\{c_{1}, \ldots, c_{R}\right\}, R \geq 2$ based on a set of training examples of the form $\left\langle\mathbf{x}_{i}, f\left(\mathbf{x}_{i}\right)\right\rangle$. The components of an instance vector $\mathbf{x}_{i}$ can take values from discrete or continuous domains. It is also possible that the values of one or more components are missing or are imprecisely recorded for certain training instances, or that an instance is mislabeled. After a classifier is built from the training data by the classifier induction algorithm, it can be used to produce labels for new instances, which have not been seen previously.

A main requirement for a good classifier is to attain high classification accuracy on unseen instances. In order to attain this goal, the classifier should generalize well over the training instances, even if this means not classifying all of them correctly. If the classifier is too closely dependent on the training instances seen, it is said to overfit the data, in which case its generalization to new examples will typically be worse.

There are additional requirements for the induced classifiers. In many practical applications there is a preference for classifiers that can be examined and interpreted easily by humans. Classifiers with low memory requirements are also typically preferred because of their generality and ease of examination. Finally, the speed of the classifier induction algorithm can be a concern, especially in applications that produce and adjust their classifiers on-line.

\section{The CLEF classifier inducer}

We present the CLEF algorithm, which constructs a $\Phi$-machine automatically by adding features as needed. As shown below, the features constructed are linearly independent, which allows the algorithm to retain the theoretical guarantees of algorithms that train $\Phi$ machines with hand-crafted features. In this section we review the details of linear machines and $\Phi$-machines, and we present in detail our feature construction method.

\subsection{Linear machines}

A linear machine is a set of $R$ linear discriminant functions $g_{i}$ used collectively to assign an instance to one of $R$ classes. Let $\mathbf{x}=\left(1, x_{1}, \ldots, x_{n}\right)$ be an instance description. Each discriminant function $g_{i}(\mathbf{x})$ has the form $\mathbf{w}_{i}^{\mathbf{T}} \mathbf{x}$, where $\mathbf{w}$ is an $(n+1)$-dimensional vector of coefficients (weights). The first element of this vector (corresponding to the input that is set to 1 for all instances) is called the bias or threshold weight. An instance is assigned class $i$ if and only if $(\forall j \neq i) g_{i}(\mathbf{x})>g_{j}(\mathbf{x})$. If a tie occurs, the instance is typically attributed 
randomly to one of the classes, but in our experiments we count such an occurrence as a failure to classify the instance correctly.

One constructs a linear machine by adjusting the weights of its discriminant functions to eliminate error, assuming that the training examples are separable. The machine starts with arbitrary initial weights, and sweeps through the set of training examples repeatedly. If an example having class $i$ is erroneously placed into class $j$, the weight vectors corresponding to the two discriminant functions for those classes are adjusted as follows:

$$
\begin{aligned}
& \mathbf{w}_{i} \leftarrow \mathbf{w}_{i}+c \mathbf{x} \\
& \mathbf{w}_{j} \leftarrow \mathbf{w}_{j}-c \mathbf{x} .
\end{aligned}
$$

The amount of correction $c$ can be computed in several different ways. In this article we use the fractional error correction rule, as described by Nilsson (1965):

$$
c=\alpha \frac{\left(\mathbf{w}_{i}-\mathbf{w}_{j}\right)^{\mathbf{T}} \mathbf{x}}{2 \mathbf{x}^{\mathbf{T}} \mathbf{x}}+\epsilon,
$$

where $\alpha \in(0,1)$ is the step size, controlling the magnitude of the correction, and $\epsilon \geq 0$ controls the "safety margin" between the two classes. Both $\alpha$ and $\epsilon$ are constants that can be tuned, automatically or by hand, for any given classification task. For the work presented here, we used fixed values of $\alpha=0.1$ and $\epsilon=0.01$ for all the classification tasks used in our experiments.

If the training instances are linearly separable, this update rule guarantees that the linear machine will converge to a boundary that classifies them correctly. If the instances are not linearly separable, this update rule will cause the weights to oscillate between different values in a given range. Several other weight update algorithms have been devised with the purpose of eliminating such oscillations. The most well known are the pocket algorithm (Gallant, 1990), the thermal perceptron rule (Frean, 1992) and the barycentric correction procedure (Poulard, 1995). However, in this article we do not adopt any of these algorithms, because we will use the weight oscillations to our advantage. We assume that weight oscillations indicate that the training examples are not separable given the current structure of our classifier, and we modify the structure by adding new features. This procedure is described in detail below.

\section{2. $\Phi$-machines}

For many tasks, linear combinations of the input values are not adequate for discriminating the groups of instances belonging to each class, because the instances are not linearly separable. When a non-linear discriminant is needed, one can resort to a $\Phi$-machine, which is a linear machine that uses features of the inputs, instead of the inputs themselves. For a $\Phi$-machine, the discriminant functions are of the form $g_{i}(\mathbf{x})=\mathbf{w}_{i}^{\mathbf{T}} \Phi_{i}(\mathbf{x})$, where $\Phi_{i}=\left\langle f_{1}, \ldots, f_{M}\right\rangle$ is a vector of linearly independent, real, single-valued functions $f_{j}: \mathbf{X} \rightarrow \Re$, independent of the weights. This means that the $f_{j}$ are not varying with the weight adjustments, which is of critical importance. It allows the error function 
of each discriminant function to have one global minimum. Thus, if the instances are linearly separable, the machine will converge to a correct separation. Multi-layered neural networks trained with backpropagation, for instance, do not satisfy this requirement, since their hidden units change with the weight adjustments. This causes the error surface to have several (usually many) local minima, and the weights can easily become trapped in such minima.

$\Phi$-machines preserve the theoretical advantages of linear machines, while allowing for non-linear combinations of the inputs. Therefore, $\Phi$-machines can represent partitions of the input space that cannot be represented by linear machines. The training procedures used for linear machines can be applied to adjust the weights of $\Phi$-machines. All the convergence theorems for linear machines apply to $\Phi$-machines, because the feature layer simply creates a different space in which the learning takes place. The $\Phi$-machine is a set of linear combinations in feature space, whereas a plain linear machine is a set of linear combinations in the input space.

Due to the great variety of classification tasks, one cannot know ahead of time which features $f_{j}$ will be useful as components of one or more discriminants. These functions $f_{j}$ should be constructed automatically, based on the training instances. A good feature construction algorithm would find features that allow the $\Phi$-machine to separate the data. We address the issue of how to construct such functions automatically in the next subsection.

Any method for automatically constructing $\Phi$-machines needs to generate functions $f_{j}$ that are linearly independent and that do not vary when the parameters $w_{j}$ of the machine are adjusted. Constructive methods that adjust the function definitions while correcting the output weights are not suitable candidates, because they generate functions $f_{j}$ that are not independent of the machine parameters $w_{j}$.

In the case of Boolean input variables, one alternative would be to choose $f_{j}$ from a set of basis functions, such as Rademacher-Walsh or Bahadur-Lazarsfeld polynomials (Duda $\&$ Hart, 1973). However, if the $f_{j}$ are orthogonal (i.e., $(\forall i \neq j) \sum_{\mathbf{x}} f_{i}(\mathbf{x}) \cdot f_{j}(\mathbf{x})=0$ and $\sum_{\mathbf{x}} f_{i}(\mathbf{x}) \cdot f_{i}(\mathbf{x}) \neq 0$ ), the information that can be gathered during training can only say whether more terms are needed but not what those terms should be. The search for a good set of discriminant functions is therefore quite difficult in this case.

\subsection{Feature construction}

We present the main technique we use for CLEF, our classifier induction algorithm, and two alternative approaches. One of these approaches is used to assess the merit of not dividing the training data. The other approach is used to assess the merit of creating features that are independent of the weights. Other feature construction methods could be used to induce a $\Phi$-machine. The only requirement is that they produce linearly independent features that are independent of the weights in the discriminant functions.

3.3.1. ELF and CLEF. The ELF function approximation algorithm (Utgoff \& Precup, 1998) constructs new features as needed by identifying subsets of instances that share intrinsic properties. The algorithm was initially developed for approximating real-valued functions. Since a $\Phi$-machine consists of a set of real-valued discriminant functions, we 
use ELF in order to construct the non-linear features of each of these functions. In this sub-section, we present ELF and the way it is incorporated in CLEF.

ELF assumes that the instances are represented using Boolean input variables. Its goal is to find features, each of which covers a set of instances that share an intrinsic property. The value of a discriminant function for an instance is computed by summing the weights for those features that are true for the instance. Let $\mathbf{X}$ be the space of all describable input instances. An ELF feature is a membership function for a subset of instances $\mathbf{X}_{j} \subseteq \mathbf{X}$ :

$$
f_{j}(\mathbf{x})= \begin{cases}1 & \text { if } \mathbf{x} \in \mathbf{X}_{j} \\ 0 & \text { otherwise }\end{cases}
$$

When a feature $f_{j}$ is multiplied by its single corresponding weight, each term $w_{j} f_{j}$ has value $w_{j}$ for the instances that $\mathbf{X}_{j}$ covers, and 0 elsewhere, thus associating a particular constituent value with a particular set of instances.

The subset $\mathbf{X}_{j}$ is represented by a pattern vector with as many components (bits) as the dimensionality of an instance vector $\mathbf{x}$. Each component of a pattern has either the value '\#' or the value ' 0 '. A '\#' matches either of the possible values of the corresponding input bit, while a ' 0 ' in the pattern matches only a ' 0 ' value. For example, the pattern ' $\# 0$ ' covers the instances ' 10 ' and ' 00 ' and does not cover either ' 01 ' or ' 11 '. The pattern of all '\#' covers every domain element because the pattern matches any domain element at every component. One pattern is strictly more general than another if and only if it covers all the instances covered by the other, and at least one additional instance as well.

Initially, each discriminant function, corresponding to each class, consists of one feature, which covers the whole instance space, and has a weight of 0 . To evaluate an instance using a discriminant function, one computes the linear combination of the feature values and feature weights. To update the approximation, the training procedure revisits the training instances and adjusts the weights of the discriminant functions using the fractional error correction rule (3). Only features that matched the instance have their weights adjusted, because features that did not match have value 0 .

For each feature, the algorithm keeps track of the errors associated with each input bit, in order to determine which feature is having the greatest difficulty in fitting. When an adjustment of the weights has ceased to be productive, the algorithm adds a new feature, which is a specialization of the feature that has been producing the largest errors. Specialization is performed by copying the feature and changing a ' $\#$ ' in its pattern to a ' 0 '. The choice of which bit to specialize is based on a form of variance of the input errors for each feature. The bit whose errors are most different from the mean bit error of the feature is specialized. The new feature will cover half of the set covered by its "parent".

The features that are created by this procedure are linearly independent. The proof of this statement can be done by induction on the number $n$ of bits that are present in an input instance. Consider the base case, in which $n=1$. The instance space contains two instances: ' 0 ' and ' 1 '. There are two features that can be defined over this instance space: the most specialized feature, which is associated with the pattern ' 0 ' and only covers the first instance, and the most general feature, which corresponds to the pattern ' $\#$ ' and covers both instances. The values of the features for each instance can be tabulated in the following 
determinant:

$$
\begin{array}{c|cc} 
& 0 & \# \\
0 & 1 & 1 \\
1 & 0 & 1
\end{array} \mid
$$

which can be reduced to a unit determinant, by subtracting the last line from the first one.

Now comes the induction step. Consider the space of the instances that can be generated by $n$ input bits. These instances can be viewed as being generated from the $(n-1)$-bit instances, by adding a ' 0 ' or a ' 1 ' on the first position of the vector. Similarly, the features that can be defined over these instances are generated from $(n-1)$-bit features by adding a ' 0 ' or a '\#' on the first position of the feature. Let $d_{n-1}$ define the determinant of the $(n-1)$-bit space input features. The determinant $d_{n}$ on the $n$-bit space can be written as:

$$
\begin{aligned}
& 0 F_{n-1} \quad \# F_{n-1} \\
& \begin{array}{l|cc}
0 X_{n-1} & d_{n-1} & d_{n-1} \\
1 X_{n-1} & 0 & d_{n-1}
\end{array} \mid
\end{aligned}
$$

The induction hypothesis is that $d_{n-1}$ can be reduced to a unit determinant. This can be done by adding and subtracting lines from each other, as we did in the base case. If there is a sequence of transformations that achieves this goal, we can apply it in the upper and lower part of $d_{n}$. The resulting determinant will have the form:

$$
\left|\begin{array}{cccc|cccc}
1 & 0 & \ldots & 0 & 1 & 0 & \ldots & 0 \\
0 & 1 & \ldots & 0 & 0 & 1 & \ldots & 0 \\
\vdots & \vdots & \ddots & \vdots & \vdots & \vdots & \ddots & \vdots \\
0 & 0 & \ldots & 1 & 0 & 0 & \ldots & 1 \\
\hline 0 & 0 & \ldots & 0 & 1 & 0 & \ldots & 0 \\
0 & 0 & \ldots & 0 & 0 & 1 & \ldots & 0 \\
\vdots & \vdots & \ddots & \vdots & \vdots & \vdots & \ddots & \vdots \\
0 & 0 & \ldots & 0 & 0 & 0 & \ldots & 1
\end{array}\right|
$$

By subtracting the bottom half of the determinant from the upper half, $d_{n}$ can also be reduced to a unit determinant. Thus, the set of all possible features is linearly independent. This means that any subset of features will be linearly independent as well.

The detailed pseudocode for CLEF is given in Algorithm 1. CLEF repeatedly samples training instances. If an instance is correctly classified, no action is taken. Otherwise, the weights of the discriminant functions are updated (as shown in the pseudocode for Algorithm 2) and, if necessary, the features are updated as well.

The process of training CLEF's classifier can be viewed as constructing a sequence of $\Phi$-machines because each time one adds a new feature, one has a new $\Phi$-machine. The previous proof ensures that at any point between two feature additions, the classifier that is 


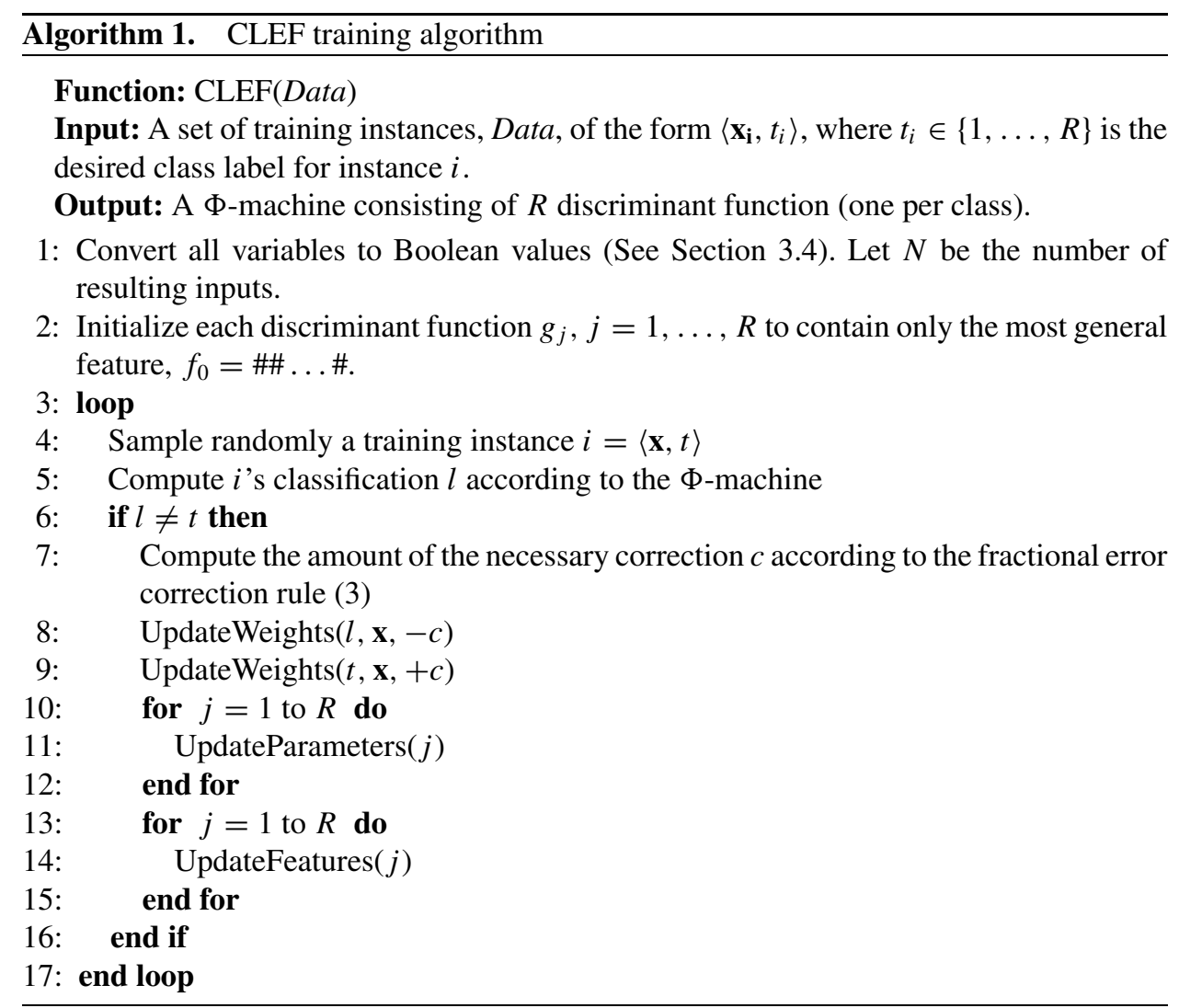

built is a $\Phi$-machine. A machine will converge to a set of weights that separates the training instances, if a separation is possible given the current set of features. If no linear separation can be found given the current feature set, by gradually reducing the size of the corrections, the weights will still settle into a particular range (Frean, 1990a). The decision of when a new feature should be added is taken by the ELF function approximator, based on an empirical measure of the stability of the weights (Utgoff, 1988). The detailed pseudocode for the feature construction process is given in Algorithm 4. In order to determine stability, the algorithm keeps track of the minimum and maximum value of the weights for each discriminant function, as well as the number of training instances seen since a new extremum was recorded. This bookkeeping is described in Algorithm 3. The weights of a discriminant function are considered stable if no new extremum was recorded for a number of training instances proportional to the current number of features in the discriminant function. The same criterion is used to determine whether the error has stabilized.

Upon adding a new feature, training resumes with a new machine. In the worst case, the process will continue until all the $2^{N}$ features that are possible have been generated. If the instances are separable when mapped through a subset of the features, they will also be 

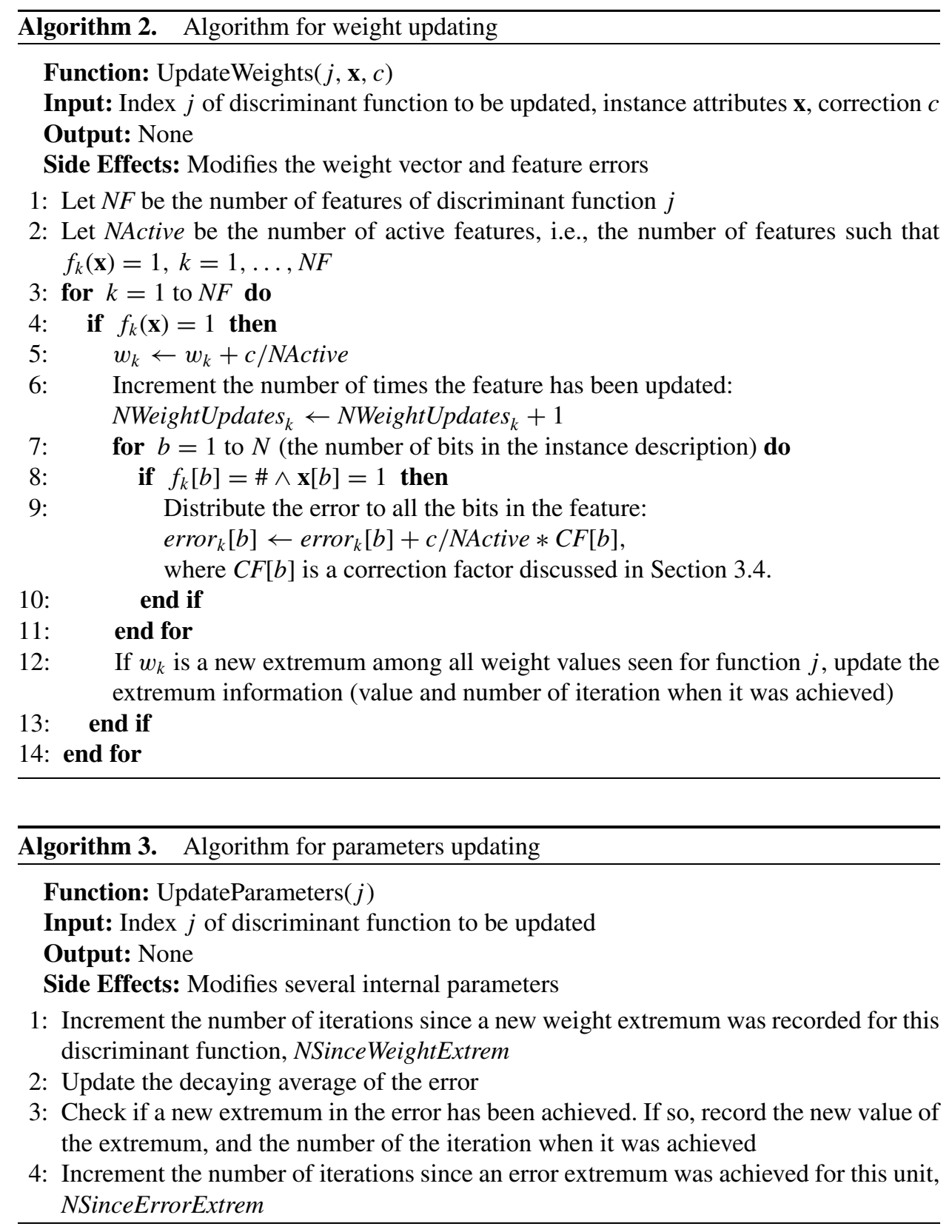

separable when the whole set is used. Thus, if a linear separation of the training instances is possible, the algorithm is guaranteed to find one.

If $N$ is the number of Boolean input variables, and $F$ is the current number of features in the algorithm, our stabilization criteria require $O(F)$ training instances to be seen before a 


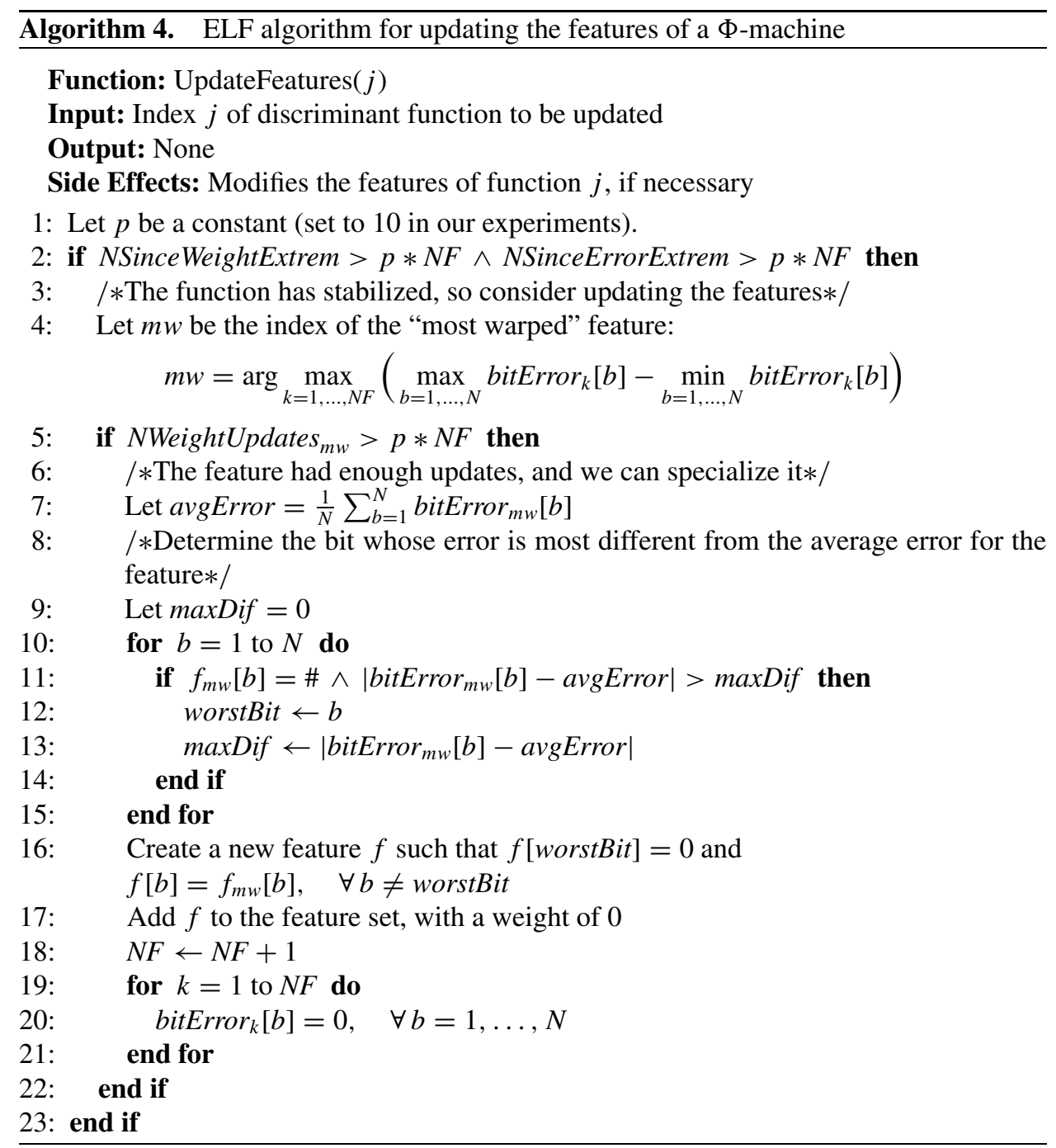

new feature can be added. In the worst case in which CLEF would create all the possible features, the time complexity of the algorithm would therefore be $O\left(1+2+3+\cdots+2^{N}-\right.$ $1)=O\left(2^{2 N}\right)$. In practice, CLEF has proven to be quite efficient with respect to the number of features it generates for a particular instance space, as shown below in Section 5. One can also consider running CLEF for a limited amount of time before adding a new feature (e.g., by looping through the training instances for a fixed number of iterations). In this case, the time complexity of the algorithm would be $O\left(2^{N} T\right)$, where $T$ is the size of the training set.

3.3.2. $\Phi$-Regression Tree $(\boldsymbol{\Phi}-\mathbf{R T})$. A variation on the previous training procedure is to define the features in a somewhat augmented pattern language. Instead of limiting a pattern 
to a string of ' 0 's and '\#'s, the string can also include ' 1 's. In this case, a subset $\mathbf{X}_{j}$ is represented by a pattern that must match exactly at ' 0 ' or at ' 1 ', but not at ' $\#$ '. A ' 0 ' in a feature matches only a ' 0 ' in an instance, a ' 1 ' matches only a ' 1 ', and a ' $\#$ ' matches a ' 0 ' or a ' 1 '. For this pattern language, the classifier constructed is equivalent to a regression tree, with one set for each path in the tree. As a result, the features are actually constructed from disjoint subsets of the data, through a process that is similar to decision tree induction. It is of interest to see how well this method fares compared to the previous one, since this would be an indication of the merit of training the features based on all the data, as opposed to disjoint subsets of decreasing size.

3.3.3. $\Phi-$ Dynamic Node Creation $(\Phi-D N C)$. Other methods of constructing features are possible if one gives up the requirement of feature independence. Of course, the resulting classifier does not have the theoretical guarantees offered by $\Phi$-machines. However, such a method still has the potential for good results in practice.

We adapted a method from the artificial neural network literature in order to generate features. Dynamic Node Creation (DNC) (Ash, 1989) is a method for generating hidden units in an artificial neural network incrementally, as needed. The idea is to start with only a bias term, and then train until the error has been minimized. At that point, if the global error is too high, a new hidden unit (with small random weights) is added to the hidden layer and the training resumes.

In our approach, a $\Phi$-machine-like structure is still used as a skeleton for the classifier.

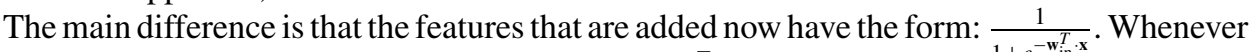
a new feature is added, its input weight vector $\mathbf{w}_{\text {in }}^{T}$ is set to 0 . The output weight for the new feature is also 0 . All the other features maintain their previous weights. The weights are updated over time using backpropagation of error. The error in this case is computed in the manner described above in Section 3.1.

The changes in the input weight vector $\mathbf{w}_{\text {in }}^{T}$ are basically changing the features used by the classifier. Therefore, this algorithm violates the $\Phi$-machine assumptions, and the resulting classifier is not a $\Phi$-machine. However, because the classifier has a form that is very similar to a $\Phi$-machine, we call this method $\Phi$-DNC.

\subsection{Input representation}

The non-linear machine described so far requires Boolean input values. Such an encoding can be generated automatically for classification tasks.

Symbolic variables are mapped into a 1-of- $m$ encoding, where $m$ is the number of possible values for each variable. A variable $v$ with possible values $v_{1}, \ldots, v_{m}$ is represented in $m$ bits. Bit $j$ will have the value 1 in an instance representation if and only if the test $v(\mathbf{x})=v_{j}$ is true.

For continuous variables, some form of discretization is needed. We have experimented with two methods for discretizing the continuous variables. The first method was suggested by Fayyad and Irani (1993). The basic mechanism is to sort the instance class labels based on the value of the continuous variable. The points at which the class label changes from one class to another are potential cutpoints for the variable. At each step, the algorithm 
looks at the list of possible cutpoints and determines the information gain for each partition generated by the cutpoint. A cutpoint is accepted if its information gain is above a certain threshold, and in this case the algorithm proceeds recursively to partition the sub-intervals left and right of the cutpoint. We found this method to be quite conservative in the number of intervals used in the discretization, which did not lead to good performance when used for our classification algorithm.

The second method was originally proposed by Fulton, Kasif, and Salzberg (1995) and then extended by Elomaa and Rousu (1996). In this case, the algorithm searches for the best split with a given upper bound $k$ on the number of intervals. The quality of a partition is evaluated by an impurity measure, and the efficiency of the search is ensured by a dynamic programming algorithm. The impurity measure used for the experiments reported in this article is information gain. We set the value of $k=\frac{N}{10}$ for all the experiments reported in this article (where $N$ is the size of the training set). We stress that $k$ is an upper bound on the number of intervals to be generated. In our experiments, most of the time the discretization process stops before $k$ is reached. Based on the intervals determined in this way, the continuous values for all the instances are transformed into a 1-of- $m$ encoding, with one bit for each of the $m \leq k$ intervals.

The time complexity of this pre-processing stage can be evaluated by examining the algorithm. The first phase requires sorting all the $N$ training instances based on the desired variable, which takes $O(N \log (N))$. The second phase is the interval extraction, during which the points at which the class label changes from one class to another are extracted (similar to Fayyad's algorithm), in $O(N)$ time. This creates $B$ potential cutoff points. In the worst case, $B=N$, but typically $B \ll N$. The third phase is the dynamic programming algorithm, which computes the impurity measure of different splits. Given a maximum number of $k$ intervals in the discretization, this algorithm has complexity $O\left(k B^{2}\right)$, as shown by Elomaa and Rousu (1996). The algorithm is applied to all the continuous variables present in the input.

We apply this pre-processing of the input variables in the same way, to all of the algorithms used in this article, so this time complexity gets added to all the algorithms analyzed below. The reason for adopting this decision was to eliminate the effect of using different representations of the data for the different algorithms. This type of discretization is an integral part of decision tree construction. For other algorithms used in the comparison, such as support vector machines and variants of DNC, such a discretization is not necessary. However, a normalization procedure is required for these algorithms if the input continuous variables have different ranges, so a pre-processing stage is unavoidable anyway.

The number of bits representing each of the input variables can vary widely. If all of the input variables are coded with the identical number of bits, then the probability of any one input bit having the value 1 is equal to any other, assuming that all the input instances are equiprobable. However, for input variables coded with a differing number of bits, even if the bits of any one variable are equiprobable, the probability of a bit being on will differ for two variables of different arity. The probability of a bit corresponding to a low arity variable being on is higher than the probability of a bit being on for a high arity variable. A simple adjustment is used to remove this bias: the error attributed to each bit is 
normalized with respect to the number of bits used to encode the variable to which the bit belongs.

The last aspect of CLEF is how it handles missing values. If the value of a variable is missing in the input then all the bits corresponding to that variable are set to 0 . This prevents the missing value from having any role in the classification process, since a pattern of all '0's will match every possible pattern. Other approaches are possible (Quinlan, 1989), such as replacing the missing value with the most likely value according to the data.

\section{Illustration of CLEF}

The Boolean encoding of the features facilitates an interpretation of the discriminant functions that form a non-linear classification machine. A human-readable interpretation can be generated automatically by printing the negation of each test for which there is a ' 0 ' in the feature's pattern.

Table 1 illustrates the features that have been constructed for one of the two discriminant functions in the hepatitis task from the UCI Machine Learning repository (Blake \& Merz, 1998). This is a two-class problem. Thus, the corresponding linear machine will have two discriminant functions, one for each class. However, due to the training procedure, these discriminant functions are always trained with equal amounts of error having opposite signs. In this two class case, the functions will always have the same features, with weights of opposite sign.

Table 1. Health test for the hepatitis task. The commas indicate boolean "and" operations.

\begin{aligned} & \hline Weight \multicolumn{1}{c}{ Feature } \\ & \hline-0.019 age $\nless 37.50 \\ & 0.013$ ascites $\neq$ no \\ &-0.012 $\begin{array}{c}\text { age } \nless 37.50, \text { liver-firm } \neq \text { yes, } \\ \text { spiders } \neq \text { no, varices } \neq \text { no }\end{array} \\ & 0.008$ intercept term \\ & 0.008 age $\nless 37.50$, protime $\nless 44.50 \\ & 0.008$ age $\nless 37.50$, varices $\neq$ no \\ & 0.007 age $\nless 37.50$, spiders $\neq$ no, varices $\neq$ no \\ &-0.006 sgot $\nless 80.50$, protime $\nless 87.50 \\ &-0.005$ steroid $\neq$ yes \\ &-0.004 bilirubin $\nless 1.35 \\ &-0.004$ protime $\nless 87.50 \\ &-0.004$ sex $\neq$ female \\ & 0.003 sex $\neq$ female, anorexia $\neq$ yes \\ &-0.002 sex $\neq$ female, liver-firm $\neq$ no \\ & 0.001 spiders $\neq$ no, histology $\neq$ yes \\ &-0.000 spiders $\neq$ no \\ & \hline\end{aligned}




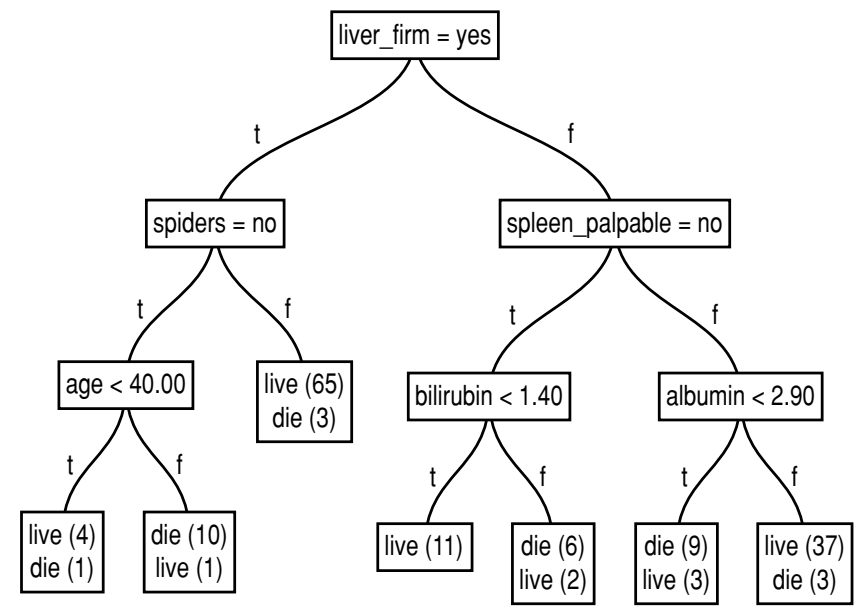

Figure 1. Decision tree for hepatitis.

This table is analogous to a "health test", which tells how to compute a score for an instance. For each line in the table, one would check whether the instance satisfies the test in the right column. If so, the corresponding weight would be added to the total score. If the total score is positive, the instance would be considered as belonging to the "die" class. For example, a patient with the following characteristics: age $=30$, ascites $=$ yes, spiders $=$ no, sex $=$ female, sgot $=79.6$, steroid $=$ no, bilirubin $=2$, protime $=80$, liverfirm $=$ yes, would evaluate to a score of $0.013+0.008-0.005-0.004=0.012$, and would therefore be classified as belonging to the "die" class.

For comparison purposes, figure 1 shows the pruned decision tree constructed by the DMTI algorithm (Utgoff, Berkman, \& Clouse, 1997) when minimizing by the minimum description length. The number in parentheses next to each class label indicates the number of examples of that class at that leaf. For a leaf with more than two classes, the classifier selects the class with the greatest number of examples. Using the same patient example, the tree classifier will push the instance down the leftmost branches of the tree and classify it as belonging to the "live" class. The purpose of the illustration is to contrast the two forms with respect to interpretability. The fact that they happen to give different answers is not important here. The issue of accuracy is discussed below.

It is not clear which of the 'health test' representation and the 'decision tree' representation is easier to interpret. One does see the 'health test' style of presentation in popular magazines from time to time, but the authors have not seen a decision tree in a similar kind of article. It seems that people do not have trouble associating positive and negative factors with different traits. In our view, one does not lose interpretability in the 'health test' format.

The only other concern regarding the interpretability of the learned classifier is that the number of features could potentially be very large. Of course, a similar concern applies to decision trees with many nodes. The results presented in the next section show that CLEF constructs fairly compact classifiers, typically with a few tens of features. In our opinion, in such classifiers one can easily look at all the features. Of course, the features are sorted 
in the order of the magnitude of their weights, so one could choose to limit the number of features shown and consider only the most important ones.

\section{Analysis}

How well does CLEF's classification accuracy compare to other classification algorithms? Does it find a separating $\Phi$-machine in a reasonable amount of time? Does it construct a large number of features, perhaps producing an incomprehensible classifier?

In order to answer these questions empirically, we compare the three algorithms presented in Section 3 with the well known decision tree algorithm C4.5 (Quinlan, 1993), with the constructive neural network training method DNC, with a simple linear machine inducer, which uses the input data directly, and with Support Vector Machines (SVM). The main idea behind DNC was briefly outlined above in Section 3.3.3.

Support Vector Machines (Vapnik, 1995) can also be viewed as constructing features automatically, and then the features are combined linearly. As in the case of $\Phi$-machines, the training is done without partitioning the data. Therefore, SVMs and CLEF are quite similar in spirit. However, for SVMs the form of the features that are constructed needs to be defined a priori. On the other hand, because SVMs do not need to compute the feature expansion of the instance, they can potentially handle high-dimensional input spaces and large numbers of constructed features better than CLEF.

In these experiments we used the SVM Matlab package created by Steve Gunn (1998). We chose a Gaussian radial basis function kernel with the global basis function width $\sigma=1.0$. We also used a tolerance to misclassification errors parameter, $C=10$. A detailed discussion of the meaning of the $C$ parameter and of ways to select it is given in Gunn (1998).

All algorithms were run on several classification tasks, mostly from the UCI Machine Learning Repository (Blake \& Merz, 1998). This allows for a comparison in terms of classification accuracy, and provides some insight on the efficiency of CLEF and the form of the function it provides.

The heart disease tasks (cleveland, switzerland, and va) are each five-class problems, whereas in many reports one sees four of the classes grouped, resulting in a two-class problem. The mplex-6 is a six-bit version of the multiplexor problem. The tictactoe data is different from the UCI task of similar name. Here, all positions that can occur during play appear as examples, labeled with 'draw', or the number of ply until a win on either side will be achieved, assuming perfect play. All positions are represented from the point of view of the player on-move, coded as ' $x$ '.

The salient difference between CLEF and decision tree inducers is that CLEF uses all the training set to construct its classifier. It should be advantageous to CLEF that it solves one classification problem using all the data, instead of many subproblems, each using only some of the data. DNC is also using all the data for training, but the features that it constructs are not linearly independent, and the algorithm has only guarantees of convergence to a local minimum. SVMs are guaranteed to converge to an optimal separating hyperplane in the feature space defined by the kernel functions. Of course, the choice of kernel functions depends on the classification task, and can greatly affect performance. 
For any task in which a training set and testing set were given, those sets were merged into a single set of examples. The tasks for which this was done were audio-no-id and soybean. In this way, ten-fold stratified cross validation could be applied to all tasks, except monks-2. For this task, we used the training and test set provided, and we did not perform cross-validation.

CLEF was trained by repeatedly sampling at random $N=100|\mathbf{X}|$ times from the training set (where $|\mathbf{X}|$ is the size of the training set), for a fixed number of epochs. Training can stop early, if the instances in the training set are perfectly separated. The same training procedure was used for DNC as well. For C4.5, the default settings were used, and pruning was turned on. CLEF does not currently use any mechanism for avoiding overfitting; the design of such a mechanism is left for future work.

When SVMs were used for multi-class classification problems, we trained classifiers that would separate each class from all the other classes. In this way, it is possible that an instance be classified as belonging to multiple classes, or to no class. Both these cases were considered as classification errors. However, such cases occurred very rarely in our experiments, so they do not have a significant effect on the results reported in Table 2.

Table 2. Accuracy results.

\begin{tabular}{|c|c|c|c|c|c|c|}
\hline Task & $\mathrm{C} 4.5$ & CLEF & $\Phi-\mathrm{RT}$ & $\Phi-\mathrm{DNC}$ & $\mathrm{DNC}$ & SVM \\
\hline audio-no-id & $77.8 \pm 6.6$ & $79.1 \pm 9.1$ & $71.5 \pm 1.2$ & $64.5 \pm 0.9$ & $79.5 \pm 1.0$ & $71.5 \pm 0.5$ \\
\hline balance-scale & $78.3 \pm 3.2$ & $92.5 \pm 4.0$ & $75.3 \pm 1.1$ & $76.3 \pm 1.1$ & $74.3 \pm 1.5$ & $78.3 \pm 1.5$ \\
\hline breast-cancer & $75.5 \pm 3.9$ & $70.3 \pm 7.1$ & $72.3 \pm 5.1$ & $66.3 \pm 4.1$ & $71.5 \pm 5.0$ & $75.5 \pm 0.1$ \\
\hline bupa & $64.6 \pm 5.6$ & $68.7 \pm 5.0$ & $65.3 \pm 5.0$ & $60.7 \pm 3.9$ & $62.7 \pm 4.0$ & $70.3 \pm 3.3$ \\
\hline cleveland & $46.8 \pm 5.4$ & $48.7 \pm 8.4$ & $48.3 \pm 6.4$ & $45.1 \pm 6.3$ & $51.7 \pm 8.8$ & $44.9 \pm 5.5$ \\
\hline hepatitis & $77.5 \pm 5.7$ & $81.9 \pm 5.2$ & $76.7 \pm 3.0$ & $79.9 \pm 5.1$ & $81.1 \pm 4.2$ & $83.1 \pm 4.2$ \\
\hline iris & $94.4 \pm 7.6$ & $94.4 \pm 7.1$ & $93.2 \pm 7.5$ & $92.5 \pm 1.5$ & $96.1 \pm 2.1$ & $96.6 \pm 2.0$ \\
\hline led24 & $76.8 \pm 4.9$ & $54.2 \pm 1.3$ & $51.2 \pm 1.1$ & $61.3 \pm 1.3$ & $66.6 \pm 1.3$ & $76.8 \pm 1.3$ \\
\hline lymphography & $28.6 \pm 2.8$ & $66.8 \pm 2.3$ & $56.5 \pm 1.3$ & $44.3 \pm 2.1$ & $42.2 \pm 1.1$ & $56.5 \pm 2.3$ \\
\hline monks-2 & $65.9 \pm 0.0$ & $88.7 \pm 0.0$ & $34.8 \pm 0.0$ & $48.1 \pm 0.0$ & $61.1 \pm 0.0$ & $90.0 \pm 0.0$ \\
\hline mplex-6 & $57.1 \pm 19.2$ & $91.4 \pm 14.6$ & $67.1 \pm 8.1$ & $45.1 \pm 9.1$ & $67.6 \pm 12.0$ & $88.8 \pm 9.8$ \\
\hline promoter & $77.3 \pm 14.2$ & $87.3 \pm 6.0$ & $79.1 \pm 5.2$ & $72.1 \pm 6.0$ & $77.3 \pm 5.0$ & $81.1 \pm 6.0$ \\
\hline soybean & $92.2 \pm 2.4$ & $91.9 \pm 3.1$ & $85.1 \pm 1.1$ & $86.9 \pm 2.0$ & $81.9 \pm 3.1$ & $91.1 \pm 2.0$ \\
\hline switzerland & $33.1 \pm 7.7$ & $35.4 \pm 14.7$ & $31.4 \pm 9.7$ & $33.4 \pm 9.7$ & $36.1 \pm 10.7$ & $39.4 \pm 5.1$ \\
\hline tictactoe & $68.1 \pm 2.3$ & $78.4 \pm 2.8$ & $68.2 \pm 1.8$ & $56.1 \pm 2.8$ & $59.1 \pm 3.1$ & $74.6 \pm 1.8$ \\
\hline va & $29.5 \pm 10.0$ & $32.9 \pm 7.2$ & $26.1 \pm 5.0$ & $29.2 \pm 6.6$ & $28.5 \pm 11.2$ & $34.1 \pm 9.8$ \\
\hline votes & $94.3 \pm 3.3$ & $94.3 \pm 3.1$ & $95.1 \pm 3.1$ & $94.4 \pm 3.3$ & $96.1 \pm 3.7$ & $96.6 \pm 3.7$ \\
\hline waveform & $70.0 \pm 10.7$ & $73.9 \pm 9.1$ & $67.0 \pm 10.1$ & $67.5 \pm 7.9$ & $69.1 \pm 11.0$ & $73.1 \pm 8.8$ \\
\hline wine & $93.3 \pm 6.0$ & $94.2 \pm 8.3$ & $93.6 \pm 8.8$ & $94.2 \pm 6.5$ & $93.3 \pm 5.5$ & $95.1 \pm 5.3$ \\
\hline zoo & $97.3 \pm 6.4$ & $96.4 \pm 4.5$ & $92.4 \pm 4.0$ & $91.0 \pm 4.5$ & $92.4 \pm 5.3$ & $95.5 \pm 5.0$ \\
\hline Average & 70.03 & 76.25 & 67.51 & 65.45 & 69.39 & 75.81 \\
\hline
\end{tabular}


Table 2 shows the accuracy results of the algorithms in terms of the mean and standard deviation for each task. All values (except for monks-2) are computed from a ten-fold stratified cross-validation, with all algorithms using the same ten partitions for each task. All algorithms used the same discretization of the input variables, except for C4.5, for which we used its built-in discretization method. We are not showing the results of the simple linear machine inducer because they are much worse than all of the other algorithms, averaging $53.2 \%$ accuracy over all the tasks. This poor performance is to be expected because most of these tasks are not linearly separable, even if the input variables are discretized.

According to a Duncan multiple range test (Walpole, 1974), CLEF and SVM are significantly better than all the other algorithms, and linear machines are significantly worse. The other algorithms are all indistinguishable from one another. Also, there is no statistically significant difference between the accuracies of CLEF and SVM. CLEF provides significantly better accuracy on average than either the decision tree inducers or the artificial neural network inducers that we tested. In addition, it is significantly more accurate on average than the CLEF variants that partition the data $(\Phi-\mathrm{RT})$ or do not construct linearly independent features $(\Phi-\mathrm{DNC})$.

The only algorithm with similar accuracy is the support vector machine inducer, which uses a similar approach of keeping the training data together and linearly combining features. The SVM features used in this case are local, covering usually just one instance. This is quite different from CLEF, which constructs very general features, covering large subsets of the instance space. Therefore, one would expect CLEF to work well when large subsets of the instance space can be weighted similarly for the purpose of classification. On the contrary, CLEF would work poorly when no such regularities exist.

Based on the empirical results, which show quite similar accuracies for SVMs and CLEF across all tasks, we hypothesize that the shape of the features constructed by CLEF is quite adequate for these tasks. The results suggest that an interesting direction for future research is using the features constructed by CLEF in a machine constructed based on the structural risk minimization principle. Of course, we would still expect a pure SVM approach to have a speed advantage over CLEF for tasks with high-dimensional input spaces, such as text categorization.

In our opinion, the classifiers produced by CLEF have an advantage over support vector machines in terms of interpretability. As shown in Table 1, CLEF generated health-test tables in which the features are described as Boolean conjunctions, and the most important features are weighted more than the others. In contrast, support vector machines produce a classification by comparing the instance to be labeled with the set of support vectors, and using a kernel function to establish the classification. When the class label depends on a few tens or hundreds of support vectors, and the kernel is a complex function (e.g., a Gaussian) it is difficult to understand the function represented by the classifier.

We gathered additional measurements for CLEF, shown in Table 3 , to indicate its time and space consumption. CPU time is in seconds, and memory cost is the total number of features in the $\Phi$-machine. Computationally, the CLEF algorithm is much more costly 
Table 3. Characteristics of the classifier produced.

\begin{tabular}{lccc}
\hline Task & CPU CLEF & Size CLEF & \% Match \\
\hline audio-no-id & $218.2 \pm 42.2$ & $88.0 \pm 2.8$ & $77.3 \pm 0.8$ \\
balance-scale & $59.9 \pm 37.8$ & $39.0 \pm 2.3$ & $66.6 \pm 1.9$ \\
breast-cancer & $191.2 \pm 8.7$ & $47.1 \pm 1.8$ & $47.7 \pm 1.6$ \\
bupa & $245.4 \pm 35.8$ & $49.2 \pm 2.4$ & $64.9 \pm 4.6$ \\
cleveland & $496.0 \pm 46.2$ & $117.4 \pm 6.1$ & $72.1 \pm 2.6$ \\
hepatitis & $58.8 \pm 18.2$ & $17.4 \pm 1.0$ & $50.4 \pm 4.9$ \\
iris & $15.4 \pm 12.8$ & $19.0 \pm 8.2$ & $74.2 \pm 6.9$ \\
led24 & $36.9 \pm 9.4$ & $76.8 \pm 4.9$ & $54.2 \pm 1.3$ \\
lymphography & $39.7 \pm 15.3$ & $28.6 \pm 2.8$ & $66.8 \pm 2.3$ \\
monks-2 & $926.2 \pm 515.1$ & $59.3 \pm 8.3$ & $27.6 \pm 2.2$ \\
mplex-6 & $0.8 \pm 0.8$ & $11.5 \pm 1.8$ & $37.6 \pm 2.2$ \\
promoter & $26.9 \pm 6.2$ & $7.8 \pm 0.4$ & $64.8 \pm 1.6$ \\
soybean & $1684.0 \pm 30.5$ & $96.9 \pm 2.5$ & $71.5 \pm 0.8$ \\
switzerland & $182.8 \pm 16.9$ & $72.2 \pm 4.5$ & $85.9 \pm 4.1$ \\
tictactoe & $5792.5 \pm 236.0$ & $241.6 \pm 14.0$ & $29.6 \pm 1.1$ \\
va & $351.2 \pm 28.8$ & $123.0 \pm 7.0$ & $70.5 \pm 1.9$ \\
votes & $22.3 \pm 1.3$ & $14.3 \pm 1.4$ & $46.3 \pm 3.4$ \\
waveform & $346.6 \pm 105.3$ & $43.6 \pm 4.9$ & $79.5 \pm 1.9$ \\
wine & $14.2 \pm 4.6$ & $16.5 \pm 3.6$ & $81.3 \pm 4.0$ \\
zoo & $3.1 \pm 0.7$ & $19.0 \pm 1.2$ & $75.8 \pm 3.0$ \\
\hline
\end{tabular}

than C4.5 (10-100 times slower). SVMs were also quite slow, but because we are using a Matlab implementation the CPU times are not directly comparable. Based on a limited CPU benchmarking, SVM training was about 10-20 times slower than C4.5. DNC's speed was comparable to the speed of CLEF. Based on this data, CLEF's running time is much better than what one would predict based on the worst-case time complexity of the algorithm. Evaluating CLEF classifiers is very fast, since it only uses Boolean matching operations and additions. The classifiers built by CLEF are about ten times faster to evaluate as neural networks built by DNC. The comparison with SVMs is again more difficult to make, especially since the speed of evaluating SVMs depends on the number of support vectors and the kernel. For the SVM choices we made, the resulting classifiers were about twice as slow to evaluate than the classifiers produced by CLEF. However, we cannot claim that this would generally be the case.

CLEF typically constructs a small set of features, each of which consists of a simple bit pattern and a single weight. In order to measure the degree of overlap of the features that form a classifier, the average percentage of features matching an instance was evaluated. The "\% Match" column shows this measure for the instances in the training set. These values show that there is a high degree of overlap in the features that are constructed. 


\section{Related work}

A variety of constructive methods have been devised for classification problems. A large class of algorithms construct networks of thresholded logic units by adding boundaries that correct for misclassified examples (Parekh, Yang, \& Honavar, 1997, 2000; Yang, Parekh, \& Honavar, 1999; Mezard \& Nadal, 1989; Nadal, 1989; Frean, 1990b). These algorithms also separate consistently labeled examples. Since these algorithms use principles that are significantly different from our approach to feature construction, we do not discuss them in detail here. The experimental results that have been published regarding these algorithms are limited, so they do not provide a good basis for comparison with CLEF. Such a detailed comparison is a worthwhile topic for future work.

Research on decision trees also focused on methods for constructing additional tests to use for tree construction. Pagallo (1990) investigated the use of Boolean combinations of the existing tests at the internal nodes of a decision trees. Several researchers (Gamma, 1999; Brodley \& Utgoff, 1995) have proposed methods for constructing linear or nonlinear combinations of input features to be used as tests at the internal nodes of a decision tree.

Several algorithms that automatically construct a neural network configuration have also been used in classification tasks. Fahlman and Lebiere's (1990) cascade correlation method constructs a new hidden unit (feature) and trains it in order to minimize the residual error. When the error stagnates, the weights of the unit are frozen. The original input variables and the newly constructed unit become the input variables for the next layer. The algorithm has produced good results when applied to classification tasks. Wynne-Jones (1992) presents an approach called node splitting that detects when the hyperplane of a hidden unit is oscillating, indicating that the unit is being pushed in conflicting directions in feature space. Such a unit is split into two units, and the weights are set so that the units are moved apart from each other along an advantageous axis. A meiosis network (Hanson, 1990) is a feed-forward network in which the variance of each weight is maintained. For a hidden unit (feature) that has one or more weights of high variance, the unit is split into two. The input weights that define the feature, and the output weight for the linear combination are altered so that the two units are moved away from their means in opposite directions.

\section{Conclusions}

In this article we presented CLEF, a new classification algorithm that constructs a $\Phi$ machine to fit multiclass data. Our algorithm constructs non-linear features as needed, and incorporates them into the discriminant functions of the $\Phi$-machine. The features are added incrementally, in a general-to-specific order. The algorithm produces a sequence of $\Phi$-machine classifiers, which will ultimately produce a classifier that separates the training instances, even if they are not linearly separable in the input variables.

The CLEF algorithm does not use the divide-and-conquer approach that is typical of decision tree induction, nor does it construct linearly dependent features, as is likely with artificial neural network induction. Our empirical results suggest that, by using all the 
training data at all times, and by constructing linearly independent features, CLEF induces classifiers that are significantly more accurate on average. CLEF's accuracy is similar to that of support vector machines, which are quite close in spirit, as discussed in Section 5. This result suggests that a combination of our feature construction method with the structural risk minimization principle could lead to superior classifiers. We argued that classifiers produced by CLEF are easier to understand than SVMs.

In the two-class case, one can view each feature constructed by CLEF as an individual binary classifier. Thus, CLEF classifiers can be viewed as ensembles, in which the decision of each member is weighted in order to decide the label of an instance. We plan to investigate further the relationship of CLEF with other ensemble methods. We also plan to carry out more empirical evaluations of CLEF, focusing on the effect of using other weight update rules and other feature construction methods.

\section{Acknowledgments}

The authors thank Margie Connell, David Aha, and three anonymous reviewers for helpful comments on previous drafts of this article. This work was supported in part by NSF grant IRI-9711239. Doina Precup also acknowledges the support of the Fulbright foundation.

Many of the UCI tasks originate from sources outside the machine learning community. The audiology data originates from Professor Jergen at Baylor College of Medicine. The breast cancer data and lymphography data come from M. Zwitter and M. Soklic of the University Medical Centre at the Institute of Oncology in Ljubljana. The bupa data was provided by Richard S. Forsyth of BUPA Medical Research Ltd. The cleveland and va data were created by Robert Detrano of the Long Beach and Cleveland Clinic Foundation. The switzerland data was produced by William Steinbrunn of the University Hospital in Zurich, and Matthias Pfisterer of the University Hospital in Basel. The remaining UCI data files not mentioned here by name were also provided to UCI by generous donors.

\section{Note}

1. To the best of our knowledge, all decision tree algorithms are based on this principle.

\section{References}

Ash, T. (1989). Dynamic node creation in backpropagation networks (Technical Report ICS Report 8901). Institute for Cognitive Science, University of California, San Diego, CA.

Blake, C., \& Merz, C. (1998). UCI repository of machine learning databases. [http://www.ics.uci.edu/ mlearn/MLRepository.html]. Irvine, CA: University of California, Department of Information and Computer Science.

Brodley, C., \& Utgoff, P. E. (1995). Multivariate decision trees. Machine Learning, 19, 45-77.

Duda, R. O., \& Hart, P. E. (1973). Pattern classification and scene analysis. New York: Wiley \& Sons.

Elomaa, T., \& Rousu, J. (1996). Finding optimal multi-splits for numerical attributes in decision tree learning (Technical Report NC-TR-96-041). NeuroCOLT.

Fahlman, S. E., \& Lebiere, C. (1990). The cascade correlation architecture. Advances in Neural Information Processing Systems (pp. 524-532). Boston, MA: MIT Press. 
Fayyad, U., \& Irani, K. (1993). Multi-interval discretization of continuous-valued attributes for classification learning. In Proceedings of the Thirteenth International Joint Conference on Artificial Intelligence (pp. 10221027). San Francisco, CA: Morgan Kaufmann.

Fisher, D. H., \& McKusick, K. (1989). An empirical comparison of ID3 and backpropagation. In Proceedings of the Eleventh International Joint Conference on Artificial Intelligence (pp. 788-793). San Francisco, CA: Morgan Kaufmann.

Frean, M. (1990a). Small nets and short paths: Optimizing neural computation. Doctoral dissertation, Center for Cognitive Science, University of Edinburgh.

Frean, M. (1990b). The upstart algorithm: A method for constructing and training feedforward neural networks. Neural Computation, 2, 198-209.

Frean, M. (1992). A thermal perceptron learning rule. Neural Computation, 4, 946-957.

Fulton, T., Kasif, S., \& Salzberg, S. (1995). Efficient algorithms for finding multi-way splits for decision trees. In Proceedings of the Twelfth International Conference on Machine Learning (pp. 244-251). San Francisco, CA: Morgan Kaufmann.

Gallant, S. I. (1990). Perceptron-based learning algorithms. IEEE Transactions on Neural Networks, 1, 179191.

Gamma, J. (1999). Discriminant trees. In Proceedings of the Sixteenth International Conference on Machine Learning (pp. 134-142). San Francisco, CA: Morgan Kaufmann.

Gunn, S. (1998). Support vector machines for classification and regression (Technical Report). ISIS, University of Southampton.

Hanson, S. J. (1990). Meiosis networks. In Advances in Neural Information Processing Systems (pp. 533-541). Boston, MA: MIT Press.

Matheus, C. J., \& Rendell, L. A. (1989). Constructive induction on decision trees. In Proceedings of the Eleventh International Joint Conference on Artificial Intelligence (pp. 645-650). San Francisco, CA: Morgan Kaufmann.

Mezard, M., \& Nadal, J. (1989). Learning feed-forward networks: The tiling algorithm. J. Phys. A: Math Gen., 22, 2191-2203.

Mooney, R., Shavlik, J., Towell, G., \& Gove, A. (1989). An experimental comparison of symbolic and connectionist learning algorithms. In Proceedings of the Eleventh International Joint conference on Artificial Intelligence (pp. 775-780). San Francisco, CA: Morgan Kaufmann.

Nadal, J. (1989). Study of a growth algorithm for a feed-forward neural network. International Journal of Neural Systems, 1, 55-59.

Nilsson, N. J. (1965). Learning machines. New York: McGraw-Hill.

Pagallo, G. M. (1990). Adaptive decision tree algorithms for learning from examples. Doctoral dissertation, University of California, Santa Cruz.

Pagallo, G. M., \& Haussler, D. (1990). Boolean feature discovery in empirical learning. Machine Learning, 5, 71-99.

Parekh, R., Yang, J., \& Honavar, V. (1997). Constructive neural network learning algorithms for multicategory real-valued pattern classification (Technical Report 97-06). Iowa State University, Computer Science Department.

Parekh, R., Yang, J., \& Honavar, V. (2000). Constructive neural network learning algorithms for pattern classification. IEEE Transactions on Neural Networks, 11(2), 436-451.

Poulard, H. (1995). Barycentric correction procedure: A fast method for learning threshold units. In Proceedings of World Congress on Neural Networks (WCNN-95) (pp. 710-713). Washington, DC.

Quinlan, J. R. (1989). Unknown attribute values in induction. In Proceedings of the Sixth International Workshop on Machine Learning (pp. 164-168). San Francisco, CA: Morgan Kaufmann.

Quinlan, J. R. (1993). C4.5: Programs for machine learning. San Mateo, CA: Morgan Kaufmann.

Rumelhart, D., Hinton, G., \& Williams, R. (1986). Learning internal representations by error propagation. In D. E. Rumelhart \& J. L. McClelland (Eds.), Parallel distributed processing: Explorations in the microstructure of cognition (vol. 1). Boston, MA: MIT Press.

Utgoff, P. E. (1988). Perceptron trees: A case study in hybrid concept representations. In Proceedings of the Seventh National Conference on Artificial Intelligence (pp. 601-606). San Francisco, CA: Morgan Kaufmann.

Utgoff, P. E., Berkman, N. C., \& Clouse, J. A. (1997). Decision tree induction based on efficient tree restructuring. Machine Learning, 29, 5-44. 
Utgoff, P. E., \& Precup, D. (1998). Constructive function approximation. In H. Motoda \& H. Liu (Eds.), Feature extraction, construction, and selection: A data-mining perspective (pp. 219-235). Boston, MA: Kluwer Academic Publishers.

Vapnik, V. (1995). The nature of statistical learning theory. Springer Verlag, New York.

Walpole, R. (1974). Introduction to statistics. Hampshire, England: MacMillan Publishers Company.

Wynne-Jones, M. (1992). Node splitting: A constructive algorithm for feed-forward neural networks. Advances in Neural Information Processing Systems (pp. 1072-1079). Boston, MA: MIT Press.

Yang, J., Parekh, R., \& Honavar, V. (1999). DistAl: An inter-pattern distance-based constructive learning algorithm. Intelligent Data Analysis, 3, 55-73.

Received May 17, 1999

Revised August 21, 2002

Accepted August 22, 2002

Final manuscript August 26, 2002 